\title{
Prediction of forming limit in DP590 steel sheet forming: An extended fracture criterion
}

\author{
Bolin $\mathrm{Ma}^{1}$, Z.G. Liu ${ }^{2}$, Zheng Jiang ${ }^{1}$, Xiangdong $\mathrm{Wu}^{1, *}$, Keshan $\mathrm{Diao}^{3}$, Min, Wan ${ }^{1}$ \\ ${ }^{1}$ School of Mechanical Engineering \& Automation, Beihang University, Beijing 100191, China \\ ${ }^{2}$ Institute of High Performance Computing, A*Star, 1 Fusionopolis Way, 138632, Singapore \\ ${ }^{3}$ State Key Laboratory of Development and Application Technology of Automotive Steels, \\ Baosteel Group, Shanghai 201900, China
}

\begin{abstract}
In this paper, we propose an extended ductile fracture criterion to study forming limit of DP 590 steel sheet. This criterion involves two independent parameters, stress triaxiality $\eta$ and Lode parameter $\mu$ base on continuum damage mechanics, and assume that the damaged and undamaged configuration obey same material plastic evolution law. Thereafter, the criterion is extended to sheet metal forming under plane stress assumption. The tensile tests and Nakajima tests are employed to investigate fracture mechanism and validate fracture criterion. The forming limit diagram (FLD) of DP590 steel sheet is obtained by Nakajima tests throughout fracture and necking features on the specimens, which represent the upper band and lower band of FLD respectively. The theoretical FLD are determined by proposed fracture criterion with left and right side of FLD respectively. The predicted FLD is in good agreement with the experimental determined FLD, which demonstrates the capability of forming limit prediction by current proposed fracture criterion.
\end{abstract}

Keywords: Ductile fracture; Stress triaxiality; Lode parameter; Forming limit; DP590 steel

\section{Introduction}

The general deformation behavior of materials can be classified as ductile or brittle depending upon capability of material undergoing plastic deformation (Dieter and Bacon, 1986).

${ }^{*}$ Corresponding author. Tel. 86-010 82338788.

Email address: wuxiangdongbuaa@163.com (Xiangdong Wu).

(C) 2016. This manuscript version is made available under the Elsevier user license http://www.elsevier.com/open-access/userlicense/1.0/ 
The fracture toughness of material, which is also called ductility, indicates the energy con5 sumption from the beginning of deformation to crack. Adequate ductility is an important engineering consideration, which allows the material to redistribute localized stresses. In the sheet forming engineering, the material ductility is usually assessed with forming limit diagram of sheet metal (Ju et al., 2015; Hussaini et al., 2015; Centeno et al., 2014; Min et al., 2014).

The forming limit diagram (FLD) first introduced by Keeler and Backofen (1964) and then extended by Goodwin (1968) has been widely used to predict fracture occurrence in sheet metal forming. Generally, the material forming limits in the forming process are predicted either by the fracture strain from simple tension test or by the limit strains based on plastic instability theory, the corresponding FLD identified by these two measured strains are fracture FLD and necking FLD respectively (Yao and Cao, 2002; Guo et al., 2013; Ma et al., $2015 \mathrm{~b})$. Nowadays, the forming limit prediction of ductile material is derived from micro-mechanical analyses of ductile fracture and continuum damage mechanics (CDM) (Panich et al., 2013; Wesenjak et al., 2016). From a micro-level perspective, the ductile fracture of a metal is a combined result of nucleation, growth and coalescence of voids (Rice and Tracey, 1969). Gurson (1977) proposed a damage mechanical model based on the observation that during plastic deformation, microvoids nucleate and grow until a local plastic neck or fracture of the intervoid matrix occurs, which causes the coalescence of neighboring voids. Gurson model is one of the first micromechanical based models for the description of ductile damage and fracture, which introduces a strong coupling between plastic strain and damage, in the presence of finite strains. However, one of the shortcomings of the Gurson model is that, whatever strain history the material might be subjected, no volume void fraction evolution will be predicted if the initial void ratio is zero. Therefore, in order to enhance the model, Tvergaard and Needleman (1984) extended and improved Gurson model by including the fitting parameters, $q_{1}, q_{2}$ and $q_{3}$, with respect to void volume fraction $f$, such that voids can nucleate depending on the strain history. This is so-called as GTN model.

On the other hand, continuum damage mechanics (CDM) study damage evolution on a macroscopic scale by introducing an internal variable, normally denoted as $D$, to quantify the microscopic damage. This simplifies the constitutive formulation and thereby the parameter calibration but neglects the microscopic damage mechanisms. Several phenomenological 35 ductile fracture criteria have been proposed in the literature. To name a few, for example, Cockcroft and Latham (1968) proposed a failure criterion by amending the equivalent plastic strain with the maximum principal stress. Johnson and Cook (1985) developed a criterion, which combined the parameters of stress triaxiality, strain rate, temperature and equivalent plastic strain. Zheng et al. (1995) introduced a ductile fracture criterion based 40 on the analyses of void shape in various stages of plastic deformation, by including a critical 
divergence ratio (VGC) to quantitatively study the nucleation, growth and coalescence of voids. Xue and Wierzbicki (2008) studied the pressure sensitivity and Lode angle dependence on damage criterion, which is experimentally validated by three point bending test for aluminum alloy 2024-T351. Bai and Wierzbicki (2008, 2010) proposed a fracture criterion contains the stress triaxiality and the Lode angle parameter based on a modification of the Mohr-Coulomb criterion. There are six parameters to be calibrated for this criterion. Lou et al. (2012) reported a fracture criterion including the effects of stress triaxiality and then further transformed the criterion to couple the Lode parameters. Recently, they modified the criterion to describe shear ductile fracture with a changeable cut-off value for the stress triaxiality (Lou et al., 2014). This criterion is validated in Al 2024-T351 for given cut-off values, and the comparison reals that this criterion is capable of describing ductile fracture behavior in wide loading conditions. These successful investigation have enhanced the insights of fracture behavior in different material systems.

However, due to either experimental calibration difficulty or applicable conditions, the fracture criterion considering stress triaxiality $\eta$ and Lode parameter $\mu$ on sheet metal forming is rarely reported. It is known that the optimal fracture criterion depends on various loading conditions. Most of criteria are based on the assumption that fracture initiates at a critical point of structure when the accumulated equivalent plastic strain with a suitable stress triaxiality weighing functions reaches a critical value (Wen, 1999). Recently, Barsoum and Faleskog (2007) have shown that the stress triaxiality measure by itself is insufficient to characterize void growth and other behavior relevant to softening and localization. Therefore, another factor, Lode parameter or Lode angle, is needed to be employed in ductile fracture studies. Together with stress triaxiality and Lode parameter, we are able to explore ductile material behavior under complex loading conditions (Nahshon and Hutchinson, 2008).

Therefore, in this work, we proposed an extended fracture criterion including stress triaxiality and Lode parameter to overcome some bottlenecks of damage mechanics in the sheet metal forming. We decompose the ductile material deformation potential into two independent parts according to damage. The fracture criterion is introduced and extended for sheet metal forming in section 2, and standardized experiment, i.e., tensile test and Nakajima test of DP590 steel specimens are performed on sheet forming machine in section 3. Based on the proposed fracture criterion, the theoretical predictions of fracture FLD of DP 590 steel are conducted and discussed in section 4. This paper concludes with a short summary and some remarks in section 5 . 


\section{Fracture criterion}

\subsection{A ductile damage model}

According to continuum damage mechanism (CDM), void evolution undergoes nucleationgrowth-coalescence mode in ductile fracture. We introduce a damage variable $\beta$ as follows

$$
\beta=\ln \left(\frac{V}{V_{0}}\right)=\ln \left(1+\frac{V_{\text {void }}}{V_{0}}\right)
$$

In incremental form,

$$
d \beta=\frac{d V}{V}=\frac{d V_{v o i d}}{V}
$$

where $V$ is the volume of macroscopic material, $V_{\text {void }}$ is the void volume, and the matrix volume is calculated by $V_{0}=V-V_{\text {void }}$. According to Rice-Traceys hypothesis (Rice and Tracey, 1969), the average strain increment of elasto- plastic material $d \varepsilon_{m}$ can be defined as

$$
d \varepsilon_{m}=\frac{1}{3} \frac{d V}{V}=\frac{1}{3} \frac{d V_{\text {void }}}{V}
$$

Generally, the void volume is relatively small in ductile material. The macroscopic ductile material law is assumed to describe as power law

$$
\Psi=k \bar{\sigma}^{m}
$$

where $\Psi$ is the yield function of material, $k$ is material constant, $\bar{\sigma}$ is the effective stress and $m$ is the exponent of yield function.

In this study, the material deformation potential decompose into two independent parts. One is the damaged part, and another part is undamaged.

\section{- Undamaged configuration}

According to conventional associated flow rule, the yield function is the plastic potential of material. The plastic law of matrix in this part is identical with the ductile yield function of macroscopic material. However, due to void defect in the material, the actual loading area is smaller than macroscopic surface. Hence, the stress state of matrix is different with that of macroscopic material. Therefore, the plastic law of matrix is defined as

$$
\Psi_{1}=k_{1} \bar{\sigma}_{1}^{m}
$$

\section{- Damaged configuration}


Generally, ductile damage is highly dependent on material ductile deformation and hydrostatic stress $\sigma_{m}$ (Rice and Tracey, 1969; Needleman, 1972). It is assumed that the damage related part of plastic potential can be studied via evolution of internal ductile damage. The plastic potential can be expressed as a function of equivalent stress $\bar{\sigma}$ and mean stress $\sigma_{m}$ : $\Psi_{d}=\Psi_{d}\left(\bar{\sigma}, \sigma_{m}\right)$. By introducing a generalized damage stress $\bar{\sigma}_{\beta}$, the plastic potential in ductile damage is described in terms of $\bar{\sigma}_{\beta}$ as

$$
\Psi_{d}=k_{\beta} \bar{\sigma}_{\beta}^{n}
$$

where $k_{\beta}$ is the material constant, $n$ is the exponent of function. The relationship of generalized damage stress $\bar{\sigma}_{\beta}$ and $\Psi_{d}$ is

$$
\bar{\sigma}_{\beta}=\left(\frac{\Psi_{d}}{k_{\beta}}\right)^{\frac{1}{n}}
$$

According to the conventional associated flow rule, by applying orthogonal rule on Eq.(6), we have

$$
\begin{aligned}
d \varepsilon_{m} & =d \lambda^{\prime} \frac{\partial \Psi_{d}}{\partial \sigma_{m}} \\
d \beta & =d \lambda^{\prime} \frac{\partial \Psi_{d}}{\partial \bar{\sigma}_{\beta}}=d \lambda^{\prime} n k_{\beta} \bar{\sigma}_{\beta}^{n-1}
\end{aligned}
$$

Substituting above equations and removing the term $d \lambda^{\prime}$, with the expression from Eq.(3) $d \beta=3 d \varepsilon_{m}$, we have

$$
\frac{1}{n} \Psi_{d}^{\left(\frac{1}{n}-1\right)} \frac{\partial \Psi_{d}}{\partial \sigma_{m}}=\frac{1}{3} k_{\beta}^{\frac{1}{n}}
$$

The integration of the above equation is

$$
\Psi_{d}^{\frac{1}{n}}=\frac{1}{3} k_{\beta}^{\frac{1}{n}} \sigma_{m}+\Psi_{d}^{\prime}(\bar{\sigma})
$$

where $\Psi_{d}^{\prime}(\bar{\sigma})$ is undetermined function, which only $\bar{\sigma}$ and constant are involved. From Eq.(7) $\Psi_{d}^{\frac{1}{n}}$ is a linear function of generalized damage stress $\bar{\sigma}_{\beta}$. Thus, according to dimensional analysis, we get: $\Psi_{d}^{\prime}(\bar{\sigma})=c_{\beta} \bar{\sigma}$. Together with above equations, the damage related part of plastic potential can be expressed as

$$
\Psi_{d}=\left(\frac{1}{3} \frac{\sigma_{m}}{\bar{\sigma}} k_{\beta}^{\frac{1}{n}}+c_{\beta}\right)^{n} \bar{\sigma}^{n}
$$

where $k_{\beta}$ and $c_{\beta}$ are material constant which is determined from experiments. If we assume 
the material follow associated flow rule, the yield function is plastic potential. Therefore, the plastic potential in each deformation increment can be describe as $\Psi$. The macroscopic material volume is defined as unit $V=1$, then matrix volume is $e^{-\beta}$ by Eq.(1), the plastic potential of matrix is $e^{-\beta} \Psi_{1}$. For the damage related part of plastic potential, we assume it distributes in whole macroscopic material. Therefore, the plastic deformation potential of material is

$$
\Psi=e^{-\beta} \Psi_{1}+\Psi_{d}=e^{-\beta} k_{1} \bar{\sigma}_{1}^{m}+k_{\beta} \bar{\sigma}_{\beta}^{n}=k \bar{\sigma}^{m}
$$

According to orthogonal rule,

$$
\begin{aligned}
& d \bar{\varepsilon}=d \lambda \frac{\partial \Psi}{\partial \bar{\sigma}}=d \lambda m k \bar{\sigma}^{m-1} \\
& d \beta=d \lambda \frac{\partial \Psi}{\partial \bar{\sigma}_{\beta}}=d \lambda\left(k_{\beta} n \bar{\sigma}_{\beta}^{n-1}-e^{-\beta} k_{1} \bar{\sigma}_{1}^{m} \frac{\partial \beta}{\partial \bar{\sigma}_{\beta}}\right)
\end{aligned}
$$

We refer to general ductile flow rule, assume $m=n$ (Wen, 1999), ignore higher-order terms, then

$$
d \beta=d \lambda k_{\beta} n \bar{\sigma}_{\beta}^{n-1}
$$

substituting above equations, the increment of damage variable $d \beta$ is expressed as

$$
\begin{aligned}
d \beta & =\frac{k_{\beta}}{k}\left(\frac{\bar{\sigma}_{\beta}}{\bar{\sigma}}\right)^{n-1} d \bar{\varepsilon} \\
& =\left(\frac{1}{3} \frac{k_{\beta}^{\frac{1}{n^{2}}}}{k^{\frac{1}{n-1}}} \frac{\sigma_{m}}{\bar{\sigma}}+c_{\beta} \frac{k_{\beta}^{\frac{1}{n}}}{k^{\frac{1}{n-1}}}\right) d \bar{\varepsilon} \\
& =\left(c_{1} \frac{\sigma_{m}}{\bar{\sigma}}+c_{2}\right)^{n-1} d \bar{\varepsilon}
\end{aligned}
$$

where, $c_{1}$ and $c_{2}$ are material constant.

$$
c_{1}=\frac{1}{3} \frac{k_{\beta}^{\frac{1}{n^{2}}}}{k^{\frac{1}{n-1}}}, \quad c_{2}=c_{\beta} \frac{k_{\beta}^{\frac{1}{n}}}{k^{\frac{1}{n-1}}}
$$

By introducing the stress triaxiality $\eta=\sigma_{m} / \bar{\sigma}$, and assuming the material obey quadratic yield function $(n=2)$ (Marciniak et al., 2002), the ductile damage can be calculated explicitly as

$$
\beta=\int_{0}^{\bar{\varepsilon}_{f}}\left(c_{1} \eta+c_{2}\right) d \bar{\varepsilon}
$$


where $\beta$ represents the damage variable, $\bar{\varepsilon}_{f}$ is the equivalent strain to fracture.

\subsection{Extended fracture criterion for sheet forming}

The above proposed ductile fracture criterion only involve in stress triaxiality $\eta$. However, the stress state of material is completely described by six independent components in terms of Cauchy stress tensor. The stress triaxiality $\eta$ measure by itself is insufficient to characterize void-induced deformation behavior, thus Lode parameter $\mu$, which plays the role of a deviatoric state parameter and is related to the third deviatoric stress invariant, has also been employed in fracture studies. We introduce $\sigma_{1}, \sigma_{2}$ and $\sigma_{3}$ as the principal stresses and Cartesian coordinate system as reference configuration. The Lode parameter $\mu$ is generally expressed as

$$
\mu=\frac{2 \sigma_{2}-\sigma_{1}-\sigma_{3}}{\sigma_{1}-\sigma_{3}}
$$

with $\sigma_{1} \geq \sigma_{2} \geq \sigma_{3}$.

In the sheet forming process, the plane stress state is usual assumed due to the extremely smaller dimension of thickness comparing with other two dimensions. The forming limit test are conducted under various strain path, which is corresponding to different fracture mechanism (Bai and Wierzbicki, 2010). It is known that there are discrepant mechanisms of voids nucleation, growth and coalescence for different stress states, which is proved by the SEM fractography of the fracture surface: the shear-induced fracture along the maximal shear stress and the dimple-dominant fracture caused by the highest principal stress (Besson, 2010). However, the void-damage evolution in different strain paths displays uniformly on meso-level. Therefore, we introduce a ratio $\tau_{\max } / \bar{\sigma}$ to reflect different mechanisms in sheet metal forming. where $\tau_{\max }$ is the maximal shear stress, which can be determined quantitatively by stress Mohr's circle.

$$
\begin{aligned}
\tau_{\max } & =\frac{\sigma_{1}-\sigma_{3}}{2} \\
\bar{\sigma} & =\sqrt{\frac{1}{2}\left[\left(\sigma_{1}-\sigma_{2}\right)^{2}+\left(\sigma_{2}-\sigma_{3}\right)^{2}+\left(\sigma_{3}-\sigma_{1}\right)^{2}\right]}
\end{aligned}
$$

Hence, substituting above equations, the ratio $\tau_{\max } / \bar{\sigma}$ can be expressed as a function of Lode parameter $\eta$ as

$$
\frac{\tau_{\max }}{\bar{\sigma}}=\frac{1}{\sqrt{3+\mu^{2}}}
$$

Barsoum and Faleskog (2007) have illustrated the physical interpretation of Lode parameter 
$\mu$ and stress triaxiality $\eta$. Together with proposed continuum damage criterion in above section, we propose a criterion which contains $\mu$ and $\eta$ as

$$
C=\int_{0}^{\bar{\varepsilon}_{f}}\left(c_{1} \eta+c_{2}\right) \frac{d \bar{\varepsilon}}{\sqrt{3+\mu^{2}}}
$$

where the fracture criterion is expressed by an accumulating parameter, here denoted as the damage indicator $C$. It is noted that the material constant $c_{1}$ and $c_{2}$ is varied slightly in different deformation types. However, in sheet forming process, it is usually assumed that these material constants are unchangeable in application.

90

\section{Experiments}

To validate the effectiveness of proposed fracture criterion in sheet metal forming, the tensile tests and the forming limit tests are carried out. The material studied in this investigation is dual-phase steel DP590, which is an advanced high strength steel (AHSS) in application. The mechanical properties of this material are tested by sheet specimens with thickness of 1.5 mm. To investigate material anisotropy, specific specimens are designed in this tensile test. Fig. 1 shows true stress - strain curves from tensile test along $0^{\circ}, 45^{\circ}$ and $90^{\circ}$ with respect to the rolling direction. It is noted that the tensile experiments under each specific direction are tested three times. This process is able to insure test accuracy and minimize man-made error in experiment. We observe this material shows slight difference of mechanical properties in three testing directions. The basic material properties are listed in Table 1 . Where, the average Lankford coefficient ( $\mathrm{R}$ value),which is a measure of the plastic anisotropy of a rolled sheet metal, is very close to $1(\bar{R}=0.958)$. For simplicity, the material anisotropy is ignored in current study. Hence, the proposed fracture criterion is applicable in this study.

In sheet metal forming, the forming limit experiments (Nakajima tests) is a widely used standard process to investigate forming limits of sheet metals (ISO, 2008). The schematic diagram of the Nakajima test operation is shown in Fig. 2. Different specimen geometries are employed to produce all possible strain states from the uniaxial tension to the balanced biaxial tension (Ma et al., 2015a). The waist widths of the specimens range from $10 \mathrm{~mm}$ to $180 \mathrm{~mm}$ with arc-shaped, which are specially designed to prevent failure from occurring at the blank holder region and to induce the failure near the center region. During the whole operation, a constant blank-holder force $(300 \mathrm{KN})$ is applied on the blank sheet, while the punch travels downward with a punch diameter of $100 \mathrm{~mm}$, and draws the blank sheet into the die cavity along constant punch speed of $0.5 \mathrm{~mm} / \mathrm{s}$. Specific lubricants are applied to the contact surfaces. The major objective is to predict failure in the forming limit experiments with a phenomenological fracture model. Therefore, for each test condition, the drawing 
Table 1. Mechanical test results of DP590 steel

\begin{tabular}{ccccccc}
\hline Thickness & Specimen label & Direction & $\sigma_{0}(\mathrm{MPa})$ & $E(\mathrm{GPa})$ & $n$ & R-value \\
\hline \multirow{3}{*}{$1.5 \mathrm{~mm}$} & DP590-0-1 & $0^{\circ}$ & 396.8 & 187.0 & 0.1754 & 0.963 \\
& DP590-0-2 & $0^{\circ}$ & 389.9 & 189.2 & 0.1775 & 0.966 \\
& DP590-0-3 & $0^{\circ}$ & 395.5 & 181.7 & 0.1779 & 0.912 \\
\hline \multirow{3}{*}{$1.5 \mathrm{~mm}$} & DP590-45-1 & $45^{\circ}$ & 402.0 & 192.6 & 0.1693 & 0.885 \\
& DP590-45-2 & $45^{\circ}$ & 403.2 & 190.4 & 0.1711 & 0.900 \\
& DP590-45-3 & $45^{\circ}$ & 401.3 & 187.1 & 0.1702 & 0.815 \\
\hline \multirow{3}{*}{$1.5 \mathrm{~mm}$} & DP590-90-1 & $90^{\circ}$ & 397.6 & 187.5 & 0.1713 & 1.089 \\
& DP590-90-2 & $90^{\circ}$ & 393.4 & 181.6 & 0.1719 & 1.096 \\
& DP590-90-3 & $90^{\circ}$ & 391.2 & 186.7 & 0.1693 & 1.143 \\
\hline
\end{tabular}

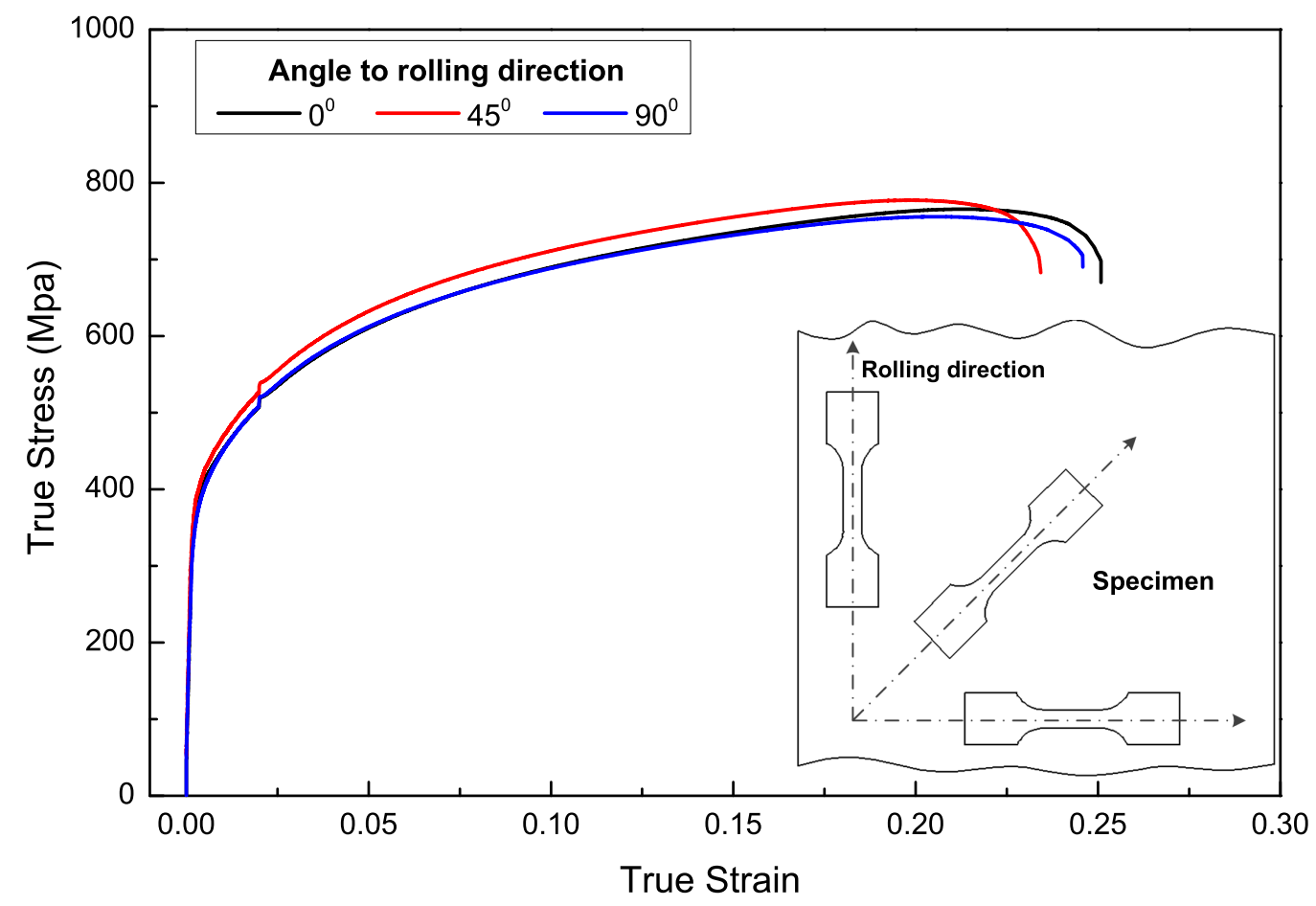

Fig. 1 The True stress-strain curves of DP590 steel by tensile test under three directions. 
depth is gradually increased until the first crack is observed on the sheet. Fig. 3 shows the deformed specimens in different fracture after Nakajima tests. The drawing depth at fracture initiation, as well as the Load - Displacement responses are recorded for each test condition.

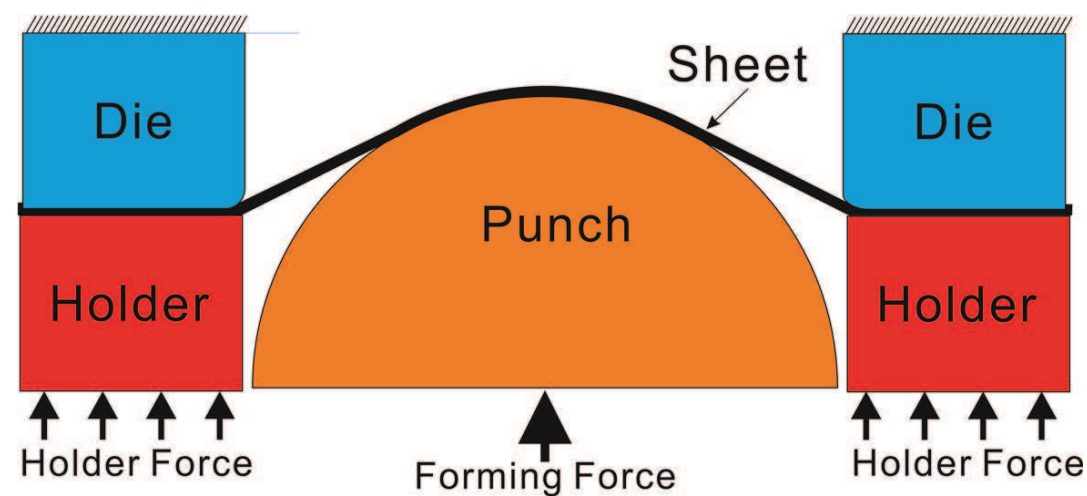

Fig. 2 The Sketch of the configurations of the Nakajima test.

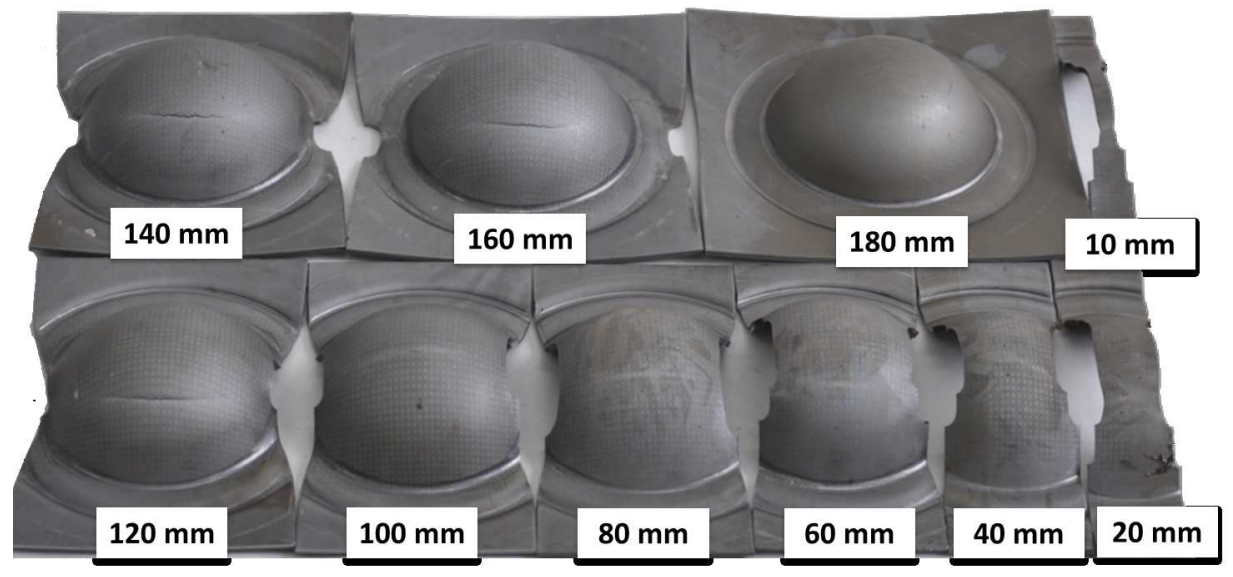

Fig. 3 Deformed specimens of DP590 steel in various test widths.

Fig.4 displays the optical strain measurement system in Nakajima tests. In this study, GMASystem optical measurement system is employed to measure the fracture limit strain from the deformation of the circles after tests. In the measurement, circular grids are electroplated to the original sheet metal with a regular spacing of $2.5 \mathrm{~mm}$ prior to the forming process. These grids follow the deformation of the part during the forming process and are maintained even in case of large relative movement between the sheet metal and the tool. The center of these grids is the reference for determining the coordinates and for the following deformation analysis. Then, the shaped component is recorded with a digital CCD camera (with a resolution of $1280 \times 1024$ pixels or $2048 \times 2048$ pixels) from various views (Fig. 4a). Photogrammetric algorithms use these images to determine the 3D coordinates of 
the dots on the sheet metal. Thus, the entire surface of the shaped sheet metal is described according to the density of the etched structure. Generally, the deformed specimen consists of distorted grids that are not assigned. Now, the system automatically creates a mesh assigning the grids to their correct neighbors. Then, in this mesh, for example each $2 \times 2$ grid field is compared to the original geometry and the corresponding surface strain tensor in space is determined. As a result, the major and minor strain and the thickness reduction of the sheet metal are available as surface information. The thickness reduction is directly calculated from the major and minor strain assuming a constant volume. The measurement accuracy of GMASystem is validated up to $2 \%$. The measured strain to fracture data in three specific loading condition, i.e., uniaxial tension, plane strain and bulge tension are listed in Table. 2. It is noted that there are two kinds of critical strains are measured, which corresponds to the deformed feature of fracture and necking respectively. The critical strains in the necking zone are directly measured from GMASystem, while the strain in the fracture zone are obtained by extrapolation of measured strains in the edge of the fracture section.
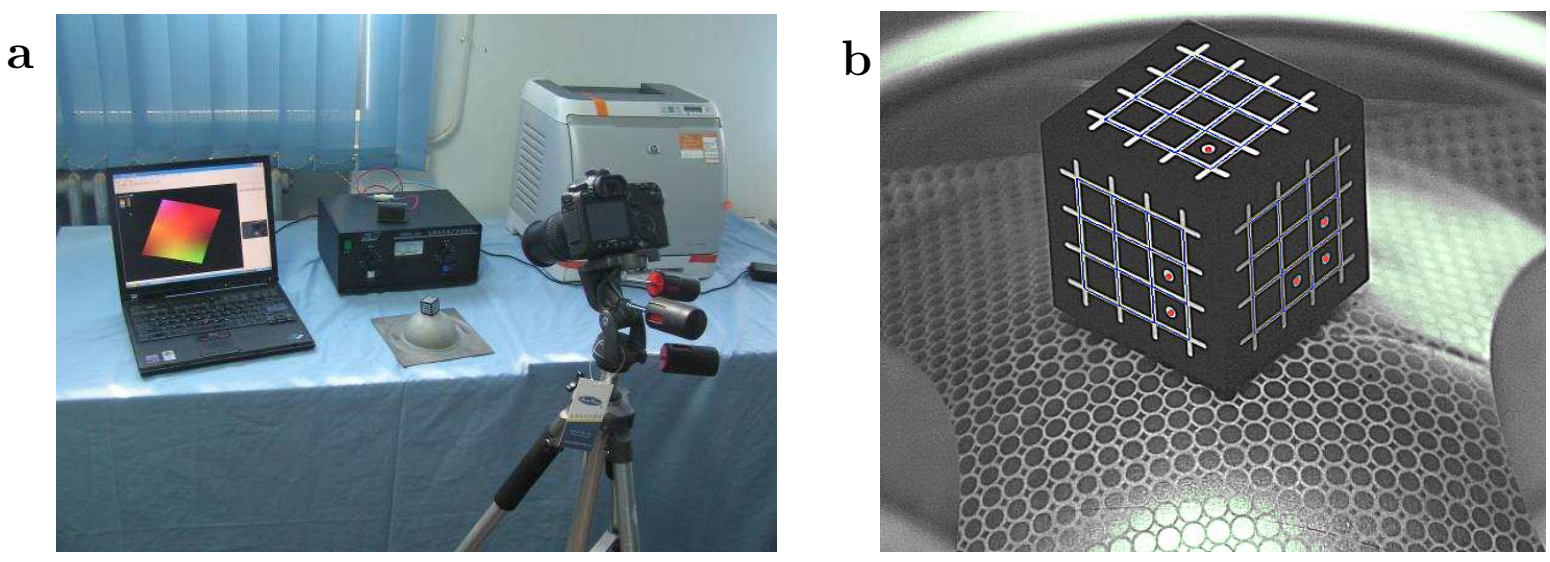

Fig. 4 The strain measurement in forming limit test. (a) The GMASystem optical strain measurement system and (b) The calibrated block in the measurement.

\section{Forming limit diagram of DP590 steel}

\subsection{Plane stress in sheet processes}

In the sheet metal forming process, a common feature is that the stress perpendicular to the surface of the sheet is small, compared with the stresses in the plane of the sheet (the membrane stress). So, the sheet is assumed in the plane stress states (Marciniak et al., 2002). We consider three principal stresses $\left(\sigma_{1}, \sigma_{2}, \sigma_{3}\right)$ applied on the sheet, in which correspond to three principal strains $\left(\varepsilon_{1}, \varepsilon_{2}, \varepsilon_{3}\right)$. Due to plane stress assumption, the stress in thickness 
Table 2. Strains-to-onset of DP590 steel under different tests

\begin{tabular}{ccccc}
\hline Test description & Strain-to-onset & Uniaxial tension & Plane strain & Bulge test \\
\hline \multirow{3}{*}{ Fracture } & $\varepsilon_{1}$ & 0.6184 & 0.4081 & 0.4574 \\
& $\varepsilon_{2}$ & -0.1985 & -0.0121 & 0.3504 \\
& $\varepsilon_{3}$ & -0.4199 & -0.3960 & -0.8078 \\
& $\bar{\varepsilon}$ & 0.6315 & 0.4645 & 0.8102 \\
\hline \multirow{3}{*}{ Necking } & $\varepsilon_{1}$ & 0.3784 & 0.2939 & 0.4206 \\
& $\varepsilon_{2}$ & -0.1504 & -0.0072 & 0.3718 \\
& $\varepsilon_{3}$ & -0.2280 & -0.2867 & -0.7924 \\
& $\bar{\varepsilon}$ & 0.3811 & 0.3352 & 0.7929 \\
\hline
\end{tabular}

direction $\sigma_{3}$ is zero. We introduce stress ratio $\alpha=\sigma_{2} / \sigma_{1}$, together with a principal strain ratio $\lambda=\varepsilon_{2} / \varepsilon_{1}$, to measure strain path variation in forming process. For a proportional process, the usual convention is to define the principal direction together with incompressible material condition $\left(\varepsilon_{1}+\varepsilon_{2}+\varepsilon_{3}=0\right)$. The deformation mode is thus:

$$
\begin{array}{ll}
\varepsilon_{2}=\lambda \varepsilon_{1}, & \varepsilon_{3}=-(1+\lambda) \varepsilon_{1} \\
\sigma_{2}=\alpha \sigma_{1}, & \sigma_{3}=0
\end{array}
$$

Thus, the mean stress $\sigma_{m}$ and equivalent stress $\bar{\sigma}$ are expressed by stress ratio as

$$
\begin{aligned}
\sigma_{m} & =\frac{1}{3}\left(\sigma_{1}+\sigma_{2}+\sigma_{3}\right)=\frac{1+\alpha}{3} \sigma_{1} \\
\bar{\sigma} & =\sqrt{\frac{1}{2}\left[\left(\sigma_{1}-\sigma_{2}\right)^{2}+\left(\sigma_{2}-\sigma_{3}\right)^{2}+\left(\sigma_{3}-\sigma_{1}\right)^{2}\right]} \\
& =\sqrt{1-\alpha+\alpha^{2}}\left|\sigma_{1}\right|
\end{aligned}
$$

Furthermore, the stress triaxiality $\eta$ and Lode parameter $\mu$ are written in terms of stress ratio $\alpha$ as

$$
\begin{aligned}
& \eta=\frac{\sigma_{m}}{\bar{\sigma}}=\frac{1+\alpha}{3 \sqrt{1-\alpha+\alpha^{2}}} \operatorname{Sgn}\left(\sigma_{1}\right) \\
& \mu=\frac{2 \sigma_{2}-\sigma_{1}}{\sigma_{1}}=2 \alpha-1
\end{aligned}
$$


Then, the strain to fracture $\bar{\varepsilon}_{f}$ is calculated in terms of principal strain ratio $\lambda$ as

$$
\begin{aligned}
\bar{\varepsilon}_{f} & =\sqrt{\frac{2}{9}\left[\left(\varepsilon_{1}-\varepsilon_{2}\right)^{2}+\left(\varepsilon_{2}-\varepsilon_{3}\right)^{2}+\left(\varepsilon_{3}-\varepsilon_{1}\right)^{2}\right]} \\
& =\frac{2}{\sqrt{3}} \sqrt{1+\lambda+\lambda^{2}}\left|\varepsilon_{1}\right|
\end{aligned}
$$

In addition, according to the hypothesis of the LevyMises Flow Rule, the mean stress $\sigma_{m}$ is unlikely to influence deformation in a solid that deforms at constant volume, while the deviatoric components are the ones associated with the shape change (Marciniak et al., 2002). This states that the ratio of the strain increments will be the same as the ratio of the deviatoric stresses.

$$
\frac{d \varepsilon_{1}}{\sigma_{1}^{\prime}}=\frac{d \varepsilon_{2}}{\sigma_{2}^{\prime}}=\frac{d \varepsilon_{3}}{\sigma_{3}^{\prime}}
$$

where $\sigma_{1}^{\prime}, \sigma_{2}^{\prime}, \sigma_{3}^{\prime}$ are three deviatoric stresses. The above equation can be integrated and expressed in terms of the true strains in a plane stress proportional process.

$$
\frac{\varepsilon_{1}}{2-\alpha}=\frac{\varepsilon_{2}}{2 \alpha-1}=\frac{\varepsilon_{3}}{-(1+\alpha)}=\frac{\lambda \varepsilon_{1}}{2 \alpha-1}
$$

From the above, the relation between the stress and strain ratio is shown as

$$
\alpha=\frac{2 \lambda+1}{2+\lambda}
$$

\subsection{FLD prediction}

In a FLD, the forming limit curve (FLC) represents the maximum major principal strains that can be reached in sheet materials at given minor principal strains prior to the onset of localized necking. Past engineering practices have shown the advantages of using FLDs in examining the failure potential, which include a good representation of material stretchability and the easiness when used for trouble shooting (Yao and Cao, 2002). It is well known that the stress states is different in the left and right sides of FLD, where tensioncompression states are shown in the lest side of FLD and tension-tension states are depicted in the right side of FLD. This conclusion is from not only experiments (Qian et al., 2003; Huang and Dong, 2006; Xie et al., 2008; Cao et al., 2015) but also finite element modeling (Ozturk and Lee, 2004; Jansen et al., 2013; Lou and Huh, 2013; Bjorklund et al., 2014). In this study, the prediction of FLD are studied with left and right side respectively by proposed ductile fracture criterion.

In simple loading condition, the proposed fracture criterion can be written by integration 
of Eq. (24) as

$$
C=\frac{c_{1} \eta+c_{2}}{\sqrt{3+\mu^{2}}} \bar{\varepsilon}_{f}
$$

There are three material constants, $c_{1}, c_{2}$ and $C$ in the ductile fracture criterion. The simple tests are carried out for sheet metals to determine these material constant, which include the uniaxial tensile test, the plane strain test and the bulge test. The strain to fracture under these three tests are denoted as $\bar{\varepsilon}_{t}, \bar{\varepsilon}_{p}$ and $\bar{\varepsilon}_{b}$ respectively. Next, the proposed ductile fracture criterion has separative form in terms of left and right side of FLD.

- 1) Left side of FLD

In this zone, the sheet are constrained in the tension-compression state $\left(\varepsilon_{1}>0, \varepsilon_{2}<0\right)$. We simply use experiment data under uniaxial tensile test $(\eta=1 / 3, \mu=-1)$ and plane strain test $(\eta=\sqrt{3} / 3, \mu=0)$ to calibrate the material constant of proposed ductile fracture criterion. Then, the fracture criterion can be expressed as

$$
\begin{aligned}
& C=\frac{c_{1}+3 c_{2}}{6} \bar{\varepsilon}_{t} \\
& C=\frac{c_{1}+\sqrt{3} c_{2}}{3} \bar{\varepsilon}_{p}
\end{aligned}
$$

Substituting the strain to fracture under uniaxial tensile test $\left(\bar{\varepsilon}_{t}=0.6315\right)$ and plane stain test $\left(\overline{\varepsilon_{p}}=0.4645\right)$ to the above equations, we have

$$
c_{1}=2.3024 C, \quad c_{2}=2.3996 C
$$

Substituting Eq.(39) to Eq.(36) and eliminate same item $C$, the fracture criterion can be determined as

$$
\bar{\varepsilon}_{f}=\frac{0.4343 \sqrt{3+\mu^{2}}}{\eta+1.0422}
$$

- 2) Right side of FLD

In this zone, the sheet are constrained in the tension-tension state $\left(\varepsilon_{1}>0, \varepsilon_{2}>0\right)$. The experiment data under plane strain test $(\eta=\sqrt{3} / 3, \mu=0)$ and bulge test are used to calibrate the material constant of proposed ductile fracture criterion. For bulge test, the stress 
triaxiality $\eta$ and Lode parameter $\mu$ are calculated by stress and strain ratio as

$$
\begin{array}{ll}
\lambda=\frac{\varepsilon_{2}}{\varepsilon_{1}}=\frac{0.3504}{0.4574}=0.7661, & \alpha=\frac{2 \lambda+1}{2+\lambda}=0.9154 \\
\eta=\frac{1+\alpha}{3 \sqrt{1-\alpha+\alpha^{2}}}=0.6647, & \mu=2 \alpha-1=0.8309
\end{array}
$$

Then, the fracture criterion are expressed as

$$
\begin{aligned}
& C=\frac{c_{1}+\sqrt{3} c_{2}}{3} \bar{\varepsilon}_{p} \\
& C=\frac{0.6647 c_{1}+c_{2}}{1.9210} \bar{\varepsilon}_{b}
\end{aligned}
$$

Substituting the strain to fracture under plane stain test $\left(\bar{\varepsilon}_{p}=0.4645\right)$ and bulge test $\left(\overline{\varepsilon_{b}}=0.8102\right)$ to the above equations, we have

$$
c_{1}=-12.7384 C, \quad c_{2}=6.0962 C
$$

Substituting Eq.(45) to Eq.(36) and eliminate same items $C$, the fracture criterion can be determined as

$$
\bar{\varepsilon}_{f}=\frac{0.0785 \sqrt{3+\mu^{2}}}{0.4786-\eta}
$$

Therefore, the fracture criterion of DP590 in both sides of forming limit prediction are determined as follows

$$
\bar{\varepsilon}_{f}= \begin{cases}\frac{0.4343 \sqrt{3+\mu^{2}}}{\eta+1.0422} & \varepsilon_{1}>0, \varepsilon_{2}<0 \\ \frac{0.0785 \sqrt{3+\mu^{2}}}{0.4786-\eta} & \varepsilon_{1}>0, \varepsilon_{2}>0\end{cases}
$$

Similarly, according to the effective critical strain under necking condition, the conservative fracture criterion of DP590 in both side of the forming limit prediction are constructed as follows

$$
\bar{\varepsilon}_{f}= \begin{cases}\frac{0.9875 \sqrt{3+\mu^{2}}}{\eta+4.8488} & \varepsilon_{1}>0, \varepsilon_{2}<0 \\ \frac{0.0234 \sqrt{3+\mu^{2}}}{0.6895-\eta} & \varepsilon_{1}>0, \varepsilon_{2}>0\end{cases}
$$

Fig. 5 shows the strain to fracture $\bar{\varepsilon}_{f}$ and stress triaxiality $\eta$ in the two branches of 
the fracture locus of DP590. It is clearly shown that the strain to fracture is not generally monotonically decreasing with triaxiality. This conclusion is consistent with the study of Bao and Wierzbicki (2004). In the study of Barsoum and Faleskog (2007), the Lode param-

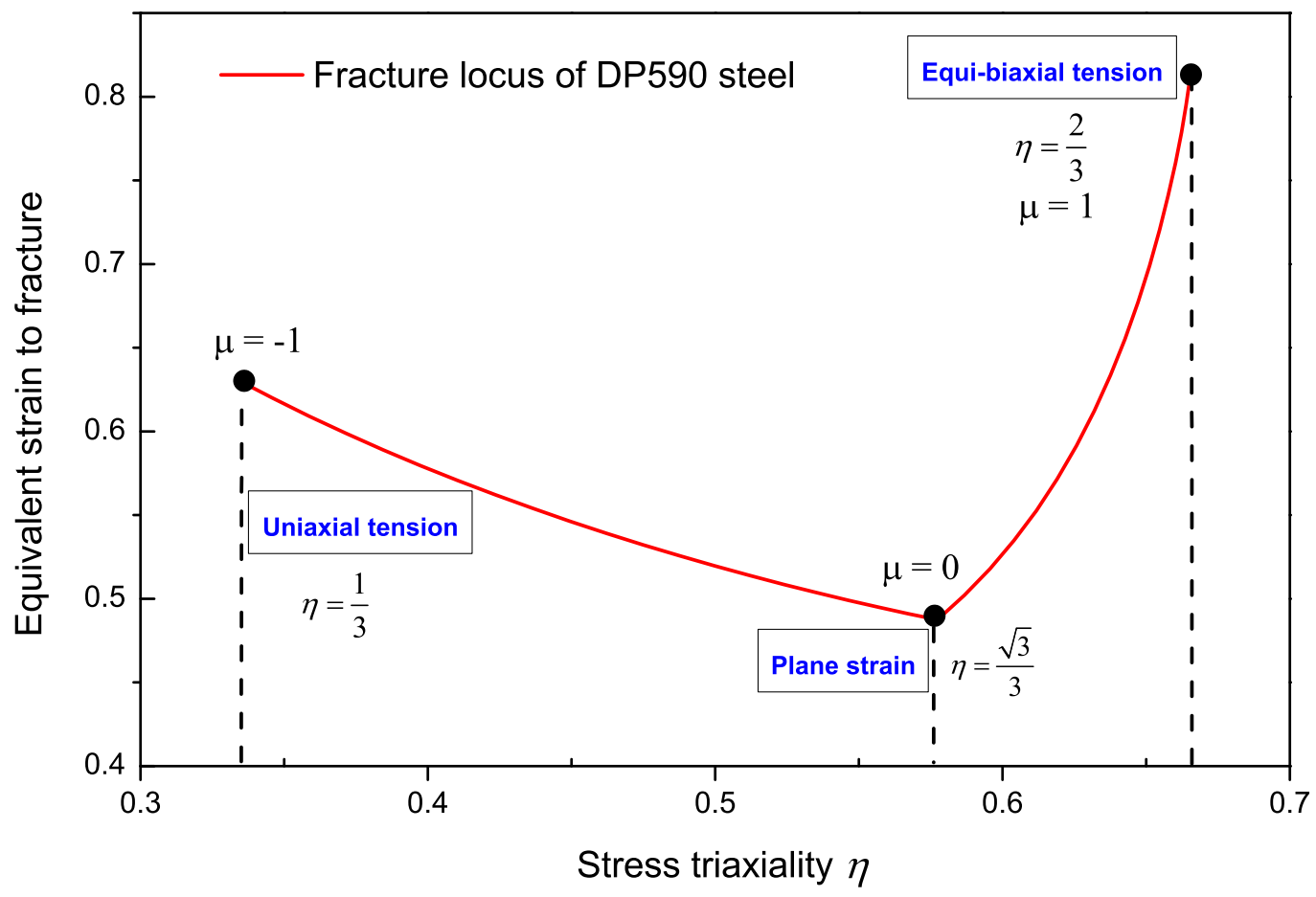

Fig. 5 Fracture locus of DP590 steel by the proposed ductile fracture criterion.

\subsection{Discussions}

In fracture mechanics, it is well known that the fracture mechanism are different in shearinduced and dimple-dominated fracture, which are corresponding to low triaxiality and high

triaxiality loading states respectively (Nahshon and Hutchinson, 2008). In this study, the fracture features of specimens are analyzed after Nakajima tests. Fig.6 shows these two fracture features in different deformation states. The shear - induced fracture occurs in 
the relatively narrow waist of arc-shaped specimen. The fracture feature displays apparent shear band with a angle of $40^{\circ}$ (Fig. 6a). While, the dimple-dominant fractures are observed in wide waist of arc-shaped specimen, where the specimen are subjected to tension-tension loading in plane stress states (Fig. 6b). It is noted that these two fracture mechanism are still challenge in fracture mechanics, the experimental observations provide a certain of evidence to fracture criterion proposed in this study.

a

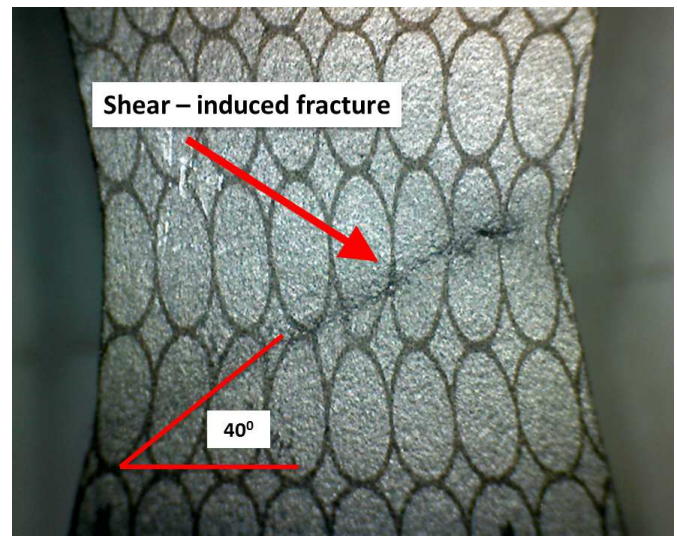

b

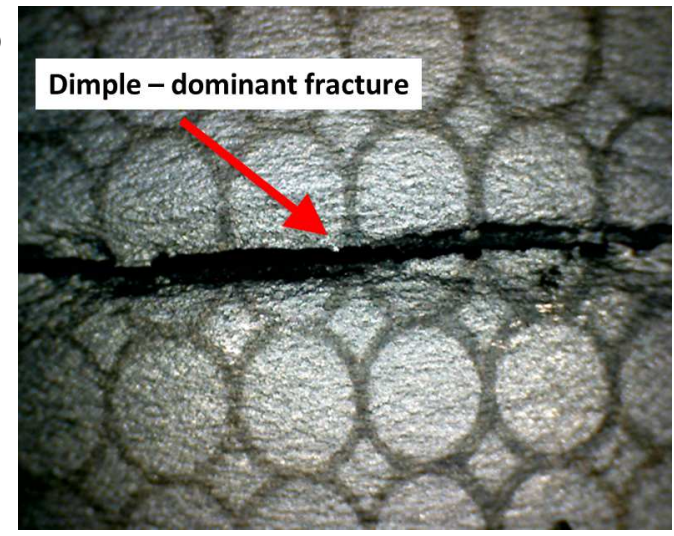

Fig. 6 The different mechanisms of ductile fracture in DP590 sheet specimen forming. (a) Shear-induced fracture (waist width of arc-shaped specimen is $10 \mathrm{~mm}$ ); (b) Dimple-dominant fracture (waist width of arc-shaped specimen is $180 \mathrm{~mm}$ ).

Fig. 7 plots the comparison of predicted FLD in DP590 sheet metal from the proposed fracture criterion, and from the Nakajima experiments. It is noted that the FLC are predicted by fracture and necking strains respectively, these two FLC represent the upper band and lower band of FLC. The key feature of the FLD is an experimentally and theoretically determined forming limit curve (FLC). The shape and location of the FLC is a characteristic of the metal, which is independent of the forming process or work piece shape. The distance between the FLC and the measured or predicted strains throughout the formed part characterizes the degree of safety. Based on current proposed fracture criterion, the forming limits of DP590 steel sheet are predicted in linear strain path. Through out the comparison, the theoretical predicted FLC exhibits a good agreement with experiment one. Thus, the prediction capability of DP590 formability is proven via current proposed fracture criterion.

\section{Conclusion}

This paper proposed a ductile fracture criterion to study forming limit of DP590 steel sheet based on the damage mechanics. This criterion involves two independent parameters, stress 


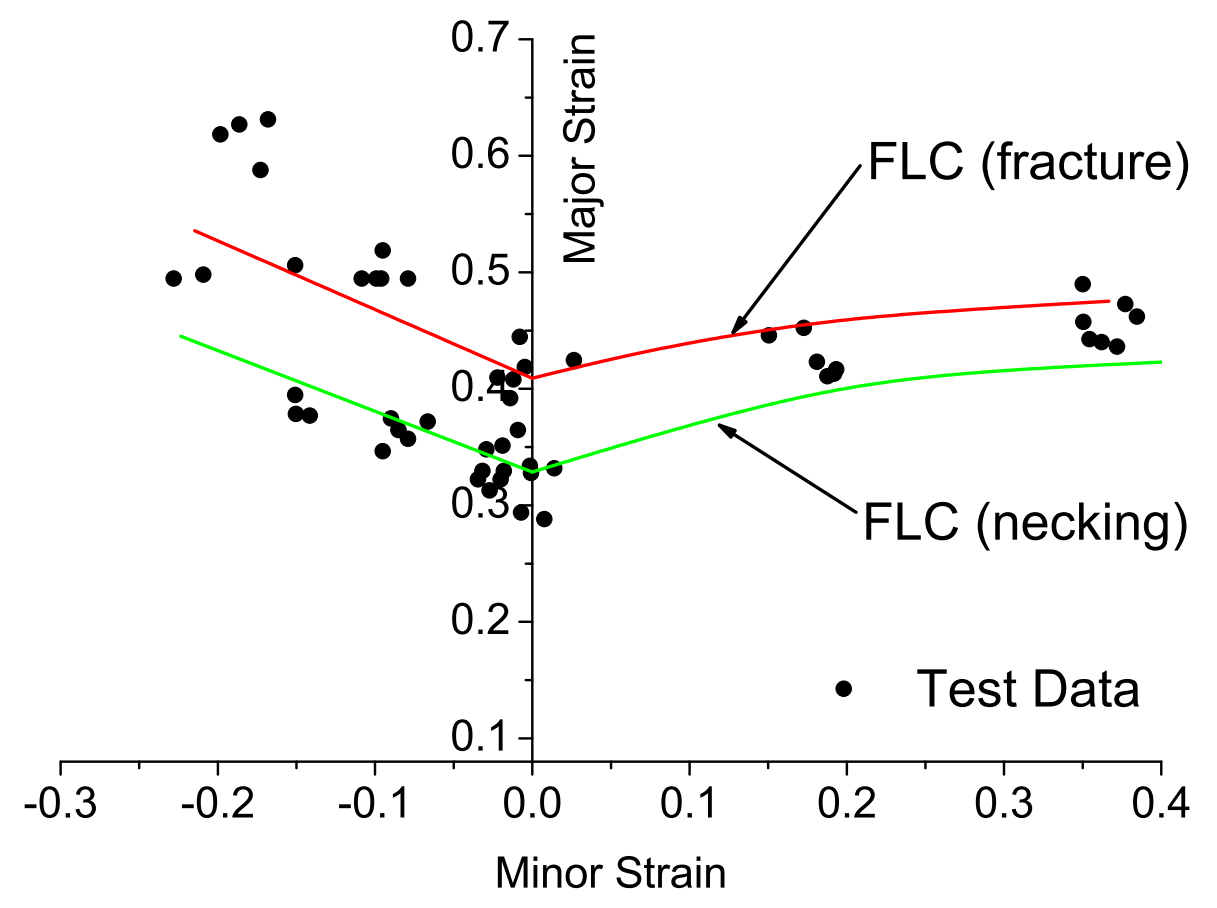

Fig. 7 Comparison of the theoretical predicted FLD with the experimental predicted one of DP590 steel.

triaxiality $\eta$ and Lode parameter $\mu$ to describe fracture strain in sheet metal forming process. The main findings were following:

(1) The fracture criterion was proposed according to continuum damage mechanism with assumptions. We assumed ductile material consist of two independent configurations, damaged and undamaged one respectively. In the undamaged configuration, the materials were described by classical plastic constitutive law. While in the damaged configuration, the material behavior was depicted by a function of plastic potential. The two parts were combined together with orthogonal rule based on an assumption that the damaged and undamaged materials obey same plastic evolution law. Thereafter, based on the experimentally and numerically study on Lode parameter, the criterion was extended to include $\eta$ and $\mu$ for sheet forming with plane stress state assumption. The fracture strain is explicitly expressed as a function of $\eta$ and $\mu$.

(2) To investigate fracture behavior and validate fracture criterion in sheet metal forming, tensile tests and Nakajima tests were employed in this study. This material shows basically isotropic property from tensile tests, which ensure the accuracy of extended assumption. To give a conservative and broad prediction of strain-to-fracture, two kinds of critical strains are measured from Nakajima tests, which correspond to the fracture and necking zone. Based on plane stress assumption, the theoretical FLD were determined by proposed fracture criterion, which was identified by three specific experiments. The criterion was written into two sides 
by experimental observation, i.e., tension-compression and tension-tension side respectively. And the theoretical predicted FLD compared with experimental determined one in good agreement, which demonstrates the capability of formability prediction via current fracture criterion.

In this study, the material fracture behavior was described by an extended fracture criterion including stress triaxiality and Lode parameter. This extension was based on experimental observation on different stress states in experiments. Actually, the fracture criterion is difficult to predict strain-to-fracture under high and low triaxiality in same form. In addition, the material is assumed as isotropy in the current study. The anisotropic material can be investigated in future.

Acknowledgments - This work was financed and supported by National Science Foundation of China (No. 51275026). The authors are also very grateful to Baosteel Co.,Ltd. for supplying sheet metals in the tests.

\section{References}

Bai, Y., Wierzbicki, T., 2008. A new model of metal plasticity and fracture with pressure and Lode dependence. Int. J. Plasticity. 24, 1071-1096.

Bai, Y., Wierzbicki, T., 2010. Application of extended Mohr-Coulomb criterion to ductile fracture. Int. J. Fract. 161, 1-20.

Bao, Y., Wierzbicki, T., 2004. On fracture locus in the equivalent strain and stress triaxiality space. Int. J. Mech. Sci. 46, 81-98.

Barsoum, I., Faleskog, J., 2007. Rupture mechanisms in combined tension and shearExperiments. Int. J. Solids Struct. 44 (6), 1768-1786.

Besson, J., 2010. Continuum models of ductile fracture: a review. Int. J. Damage Mech. 19, $3-52$.

Bjorklund, O., Govik, A., Nilsson, L., 2014. Prediction of fracture in a dual-phase steel subjected to non-linear straining. J. Mater. Process. Tech. 214, 2748-2758.

Cao, T. S., Bobadilla, C., Montmitonnet, P., Bouchard, P. O., 2015. A comparative study of three ductile damage approaches for fracture prediction in cold forming processes. J. Mater. Process. Tech. 216, 385-404.

Centeno, G., Bagudanch, I., Martnez-Donaire, A. J., Garca-Romeu, M. L., Vallellano, C., 2014. Critical analysis of necking and fracture limit strains and forming forces in singlepoint incremental forming. Mater. Design 63, 20-29. 
Cockcroft, M. G., Latham, D. J., 1968. Ductility and the workability of metals. J. Inst. Metals 101, 33-39.

Dieter, G. E., Bacon, D., 1986. Mechanical metallurgy. New York: McGraw-Hill.

Goodwin, G. M., 1968. Application of strain analysis to sheet metal forming problems in the press shop. SAE paper No. 680093.

Guo, J., Liao, B., Liu, L. G., Li, Q., Ren, X. J., Yang, Q. X., 2013. Forging limit of a novel high-speed-steel cold work roll based on ductile fracture criteria by finite element model. Mater. Design 52, 1027-1034.

Gurson, A. L., 1977. Continuum of ductile rupture by void nucleation and growth-Part I: Yield criteria and flow rules for porous ductile media. J. Eng. Mater. Tech. 99, 2-15.

Huang, J. K., Dong, X. H., 2006. Research progress of meso-damage mechanics for ductile fracture criterion in metal forming. J. Shanghai Jiaotong Univ. (Engl. Ed.) 40 (10), 17481753.

Hussaini, S. M., Krishna, G., Gupta, A. K., Singh, S. K., 2015. Development of experimental and theoretical forming limit diagrams for warm forming of austenitic stainless steel 316 . J. Manuf. Process 18, 151-158.

ISO, 2008. Metallic materials sheet and strip determination of Forming Limit Curves. Part 2: Determination of Forming Limit Curves in the Laboratory. Austrian Standards Institute, Wien, 12004-2:2008.

Jansen, Y., Loge, R., Milesi, M., Massoni, E., 2013. An anisotropic stress based criterion to predict the formability and the fracture mechanism of textured zinc sheets. J. Mater. Process. Tech. 213, 851-855.

Johnson, G. R., Cook, W. H., 1985. Fracture characteristics of three metals subjected to various strains, strain rates, temperatures and pressures. Eng. Fract. Mech 21 (1), 31-48.

Ju, L., Mao, T., Li, H., 2015. An experimental and numerical study of forming limits of AA5182-O. Int. J. Adv. Manuf. Tech. 79, 221-228.

Keeler, S., Backofen, W., 1964. Plastic instability and fracture in sheets stretched over rigid punches. ASM Trans. Quart. 56, 25-48.

Lou, Y. S., Huh, H., 2013. Prediction of ductile fracture for advanced high strength steel with a new criterion: Experiments and simulation. J. Mater. Process. Tech. 213, 1284-1302.

Lou, Y. S., Huh, H., Lim, S., Pack, K., 2012. New ductile fracture criterion for prediction of fracture forming limit diagrams of sheet metals. Int. J. Solids Struct. 49, 3605-3615.

Lou, Y. S., Yoon, J. W., Huh, H., 2014. Modeling of shear ductile fracture considering a changeable cut-off value for stress triaxiality. Int. J. Plasticity 54, 56-80. 
Ma, B., Yuan, W., Wu, X., Li, X., Wan, M., 2015a. Study on temperature distribution of HSS in hot FLD test. Appl. Therm. Eng. 89, 144-155.

Ma, X., Li, F., Li, J., Wang, Q., Yuan, Z., Fang, Y., 2015b. Analysis of forming limits based on a new ductile damage criterion in St14 steel sheets. Mater. Design 68, 134-145.

Marciniak, Z., Duncan, J. L., Hu, S. J., 2002. Mechanics of sheet metal forming. ButterworthHeinemann, USA.

Min, J. Y., Lin, J. P., Li, J. J., 2014. Forming limits of mg alloy ZEK100 sheet in preform annealing process. Mater. Design 53, 947-953.

Nahshon, K., Hutchinson, J. W., 2008. Modification of the gurson model for shear failure. Euro. J. Mech/A: Solids 27, 1-17.

Needleman, A., 1972. Void growth in an elastic-plastic medium. J. Applied Mech. 39, 964970.

Ozturk, F., Lee, D., 2004. Analysis of forming limits using ductile fracture criteria. J. Mater. Process. Tech. 147, 397-404.

Panich, S., Barlat, F., Uthaisangsuk, V., Suranuntchai, S., Jirathearanat, S., 2013. Experimental and theoretical formability analysis using strain and stress based forming limit diagram for advanced high strength steels. Mater. Design 51, 756-766.

Qian, L. Y., Fang, G., Zeng, P., Wang, Q., 2003. Experimental and numerical investigations into the ductile fracture during the forming of flat-rolled 5083-O aluminum alloy sheet. J. Mater. Process. Tech. 220, 264-275.

Rice, J. R., Tracey, D. M., 1969. On the ductile enlargement of voids in triaxial stress field. J. Mech. Phys. Solids 17, 201-217.

Tvergaard, V., Needleman, A., 1984. Analysis of the cup-cone fracture in a round tensile bar. Acta Metall. 32 (1), 157-169.

Wen, T., 1999. Research on ductile damage model and its application in metal plastic processing. Chongqing: Chongqing University.

Wesenjak, R., Krempaszky, C., Werner, E., 2016. Prediction of forming-limit curves of dualphase steels based on a multiple length scale modelling approach considering material instabilities. Comp. Mater. Sci. 111, 277-288.

Xie, Y. M., Yin, S. R., Luo, Z. Z., Pan, Y. S., 2008. The application of ductile criterions in the forming process of advanced high strength steel sheet. J. Eng. Design 15 (4), 283-289.

Xue, L., Wierzbicki, T., 2008. Ductile fracture initiation and propagation modeling using damage plasticity theory. Eng. Fract. Mech. 75, 3276-3293.

Yao, H., Cao, J., 2002. Prediction of forming limit curves using an anisotropic yield function with prestrain induced backstress. Int. J. Plasticity 18, 1013-1038. 
Zheng, C. Q., Zhou, L., Zhang, K. S., 1995. Research on micromechanics for ductile damage and its application for metals. Beijing: National Defense Industry Press. 


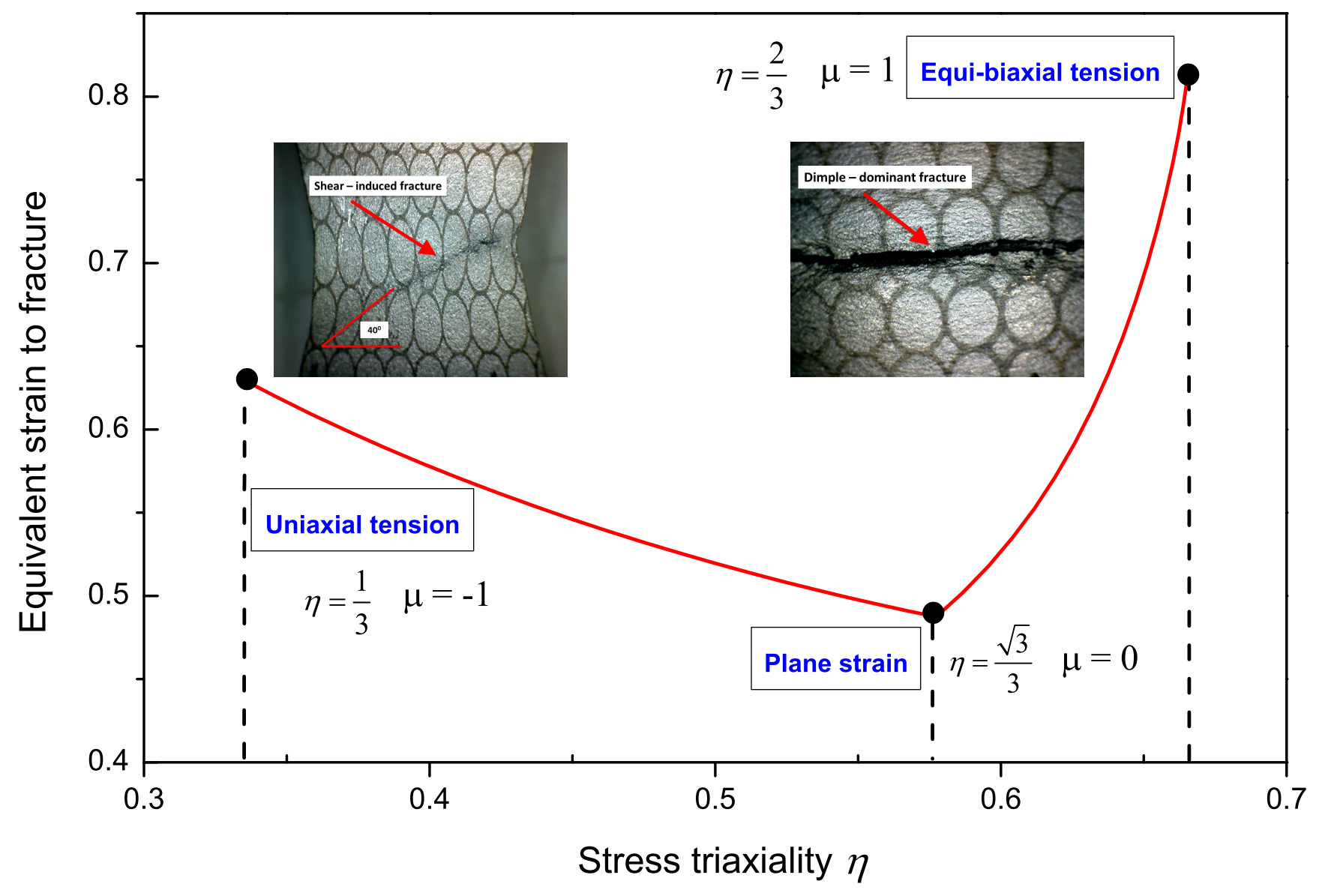

